\title{
A Model with Traffic Routers, Dynamically Managing Signal Phases to Address Traffic Congestion in Real Time
}

\author{
Rajendra S. Parmar ${ }^{1}$, Bhushan Trivedi ${ }^{2}$, Aleksandar Stevanovic ${ }^{3}$ \\ ${ }^{1}$ Gujarat Technological University, Ahmedabad, India \\ ${ }^{2}$ GLS Institute of Computer Technology, Ahmedabad, India \\ ${ }^{3}$ Florida Atlantic University, Boca Raton, FL, USA \\ Email: raj@emerging-india.com
}

How to cite this paper: Parmar, R.S., Trivedi, B. and Stevanovic, A. (2018) A Model with Traffic Routers, Dynamically Managing Signal Phases to Address Traffic Congestion in Real Time. Journal of Transportation Technologies, 8, 75-90. https://doi.org/10.4236/jtts.2018.81005

Received: November 16, 2017

Accepted: January 9, 2018

Published: January 12, 2018

Copyright $\odot 2018$ by authors and Scientific Research Publishing Inc. This work is licensed under the Creative Commons Attribution International License (CC BY 4.0).

http://creativecommons.org/licenses/by/4.0/

\begin{abstract}
On-road Vehicular traffic congestion has detrimental effect on three lifelines: Economy, Productivity and Pollution (EPP). With ever increasing population of vehicles on road, traffic congestion is a major challenge to the economy, productivity and pollution, notwithstanding continuous developments in alternative fuels, alternative sources of energy. The research develops accurate and precise model in real time which computes congestion detection, dynamic signaling algorithm to evenly distribute vehicle densities while ensuring avoidance of starvation and deadlock situation. The model incorporates road segment length and breadth, quality and achievable average speed to compute road capacity. Vehicles installed with GPS enabled devices provide their location, which enables computing road occupancy. Road occupancy is evaluated based on number of vehicles as well as area occupied by vehicles. Ratio of road occupancy and road capacity provides congestion index important to compute signal phases. The algorithm ensures every direction is serviced once during a signaling cycle ensuring no starvation. Secondly, the definition of minimum and maximum signal timings ensures against dead lock situation. A simulator is developed to validate the proposition and proves it can ease congestion by more than $50 \%$ which is better than any of the contemporary approaches offering $15 \%$ improvement. In case of higher congestion index, alternate routes are suggested based on evaluation of traffic density graphs for shortest route or knowledge database. The algorithm to compute shortest route is optimized drastically, reducing computation cost to $3 * \sqrt{2} N$ vis-à-vis computation cost of $N^{z}$ by classical algorithms. The proposal brings down the cost of implementation per traffic junction from USD 30,000 to USD 2000.
\end{abstract}




\section{Keywords}

Dynamic Traffic Assignment, Intelligent Transportation Systems, Intelligent Vehicles, Road Traffic Control, Road Traffic Sensing, Traffic Management, Vehicle Detection, Vehicle Routing, Traffic Signals, Vehicular Congestion Detection System, Vehicular Traffic, Vehicle Mobility Sensors

\section{Introduction}

Approaches to vehicle detection systems employed conventional inroad sensor leveraging technologies like infrared, ultra sound, magnetism and video streaming. These technologies are not cost-effective from installation and maintenance aspects; have poor accuracy and repeatability; require space and power. Thereafter, Adhoc networks like VANET, MANET and connected vehicles were evaluated. It suffers from poor reliability. Contemporary approaches deploy GPS, A-GPS, smart phones, wireless communication and big data solutions. Analytical, mathematical and statistical models were attempted to understand and build predictive, preemptive systems to annihilate congestion.

This paper proposes real time data acquisition to address vehicular traffic congestion employing in-vehicle GPS system, traffic routers, traffic servers, big-data incorporating infrastructure data, knowledge system for travel history \& behavioral history, high end computing engine and communication network.

The paper has 5 sections. Section 2 is literature survey to discuss research and developments undertaken in this area. Section 3 is the proposition defining strategies of assigning traffic signal phases. Priority is given to a direction with largest cumulative waiting time of vehicles. Area of the vehicle is considered to compute road occupancy and compared with road capacity to calculate congestion index. The data enables computation of road occupancy ahead, to determine how many vehicles can be accommodated in the road segment ahead. Rules are defined to ensure no deadlock situation occurs while assigning signal phases. The sequential-cyclic assignment of signal phases ensures there is no signal phase starvation. The overall topology of the solution is discussed with vehicle detection, vehicle router and traffic server. Section 4 describes analyses, results and conclusions. A simulator is developed to analyze different scenarios like: 1) Single direction: fixed signal phase; 2) Traffic junction: Fixed signal phases; 3) Traffic junction: Dynamic signal phases clearly demonstrating congestion reduction by more than $50 \%$.

Section 5 suggests future work to accurately monitor emission from vehicles for global warming, possible traffic signal avoidance, post accident analyses, monitoring road quality, tax collections based on pay-per-use and capturing traffic offenses.

\section{Literature Research \& Review}

The problem was addressed by using acceleration data from undedicated mobile 
phones [1]. This approach consumes lower power compared to GPS based technologies. The paper addresses lacuna of employing dedicated in-road sensors, the other challenges are 1) Too much data acquisition as vehicle acceleration changes frequently. 2) Dependency on cellular data; 3) Multi-cellular operator in a city demands multi-agency coordination for data collation.

The active phones in cellular network generate CDRs (Call Data Record) where as the idle phones do not generate CDR [2]. The paper studied the data set obtained over a period of month to arrive at congestion pattern. However, dependency on cellular service providers poses administrative and coordination challenges.

Researchers employed a simulator based approach. It generates traffic conditions by assigning random traffic densities with predefined maxima and minima values [3]. The prediction errors are $2 \%$ to $12 \%$ for a 5 to 30 minutes window respectively.

The paper employs IEEE 802.11 network beacon frames sent periodically [4]. To detect the vehicle and its location, a smart phone with IEEE 802.11 is used which detects the frame even at low power. It derives its name COTraMS as it is collaborative and opportunistic since it derives information from IEEE 802.11 network. The approach eliminates usage of GPS technology to save power, however road side sensors offer far too many operational disadvantages.

The paper discovers traffic bottle necks in spatiotemporal coordinates (Spatial-Location; Temporal-Time) [5]. The sensing is done through location based services. The data acquired is cleansed to indentify vehicles, compute vehicle speed, location and determine journey path. Based on this information congestion locations are discovered. Once again limitation of dependencies on cellular agency is a big challenge.

The paper banks on Localized Handling Network Event Systems (LocHNESs) platform developed by Telecom Italia [6]. The proposed system takes inputs from fixed sensors as well as GPS enabled devices. The localization engine determines the location of the vehicle and tracking filter finds out the trajectory of the vehicle from latitude and longitude thus computing speed of the vehicle. Short calls lasting for a very short duration are ignored to reduce data over load. Traffic map application produces a raster map, generating the network. It continues to offer operational challenges because of fixed sensors and problems associated to tie-up with multi-telecom service providers.

The paper detects the spare road capacity ahead of the vehicles to check if the road ahead can accommodate additional vehicles [7]. This is termed as AFRC (Available Forward Road Capacity). The exit detector detects the buffer, if found full it communicates accordingly. The AFRC reduced the delay by $12 \%$ over commercially available systems like SCATS.

Conventional sensor technologies require different mounting methodology have diverse operating principles and implementation criterion [8]. The survey paper compares conventional sensors and infers GPS is the most accurate tech- 
nology.

The above literature review is summarized in Table 1.

\section{Proposition}

The proposed architecture is presented in Figure 1 depicting vehicles, traffic routers, traffic server and wireless communication. The modules incorporated in the traffic server are road infrastructure database, vehicle detection, computing traffic signal phases, traffic router or/traffic signal driver, traffic Server/computing engine, alternate routes and knowledge system.

Table 1. Summary of literature review.

\begin{tabular}{|c|c|c|}
\hline Principle & Advantage & Disadvantage \\
\hline $\begin{array}{c}\text { Undedicated Mobile } \\
\text { Phones }\end{array}$ & $\begin{array}{l}\text { 1) Relies on acceleration } \\
\text { 2) Energy Efficient } \\
\text { 3) Measures speed, congestion }\end{array}$ & $\begin{array}{l}\text { 1) Accelerometer } \\
\text { measurements prone to } \\
\text { inaccuracies, erroneous data } \\
\text { 2) Depends on multiple cellular } \\
\text { service providers for } \\
\text { location detection }\end{array}$ \\
\hline $\begin{array}{l}\text { Anonymized } \\
\text { Signaling }\end{array}$ & $\begin{array}{l}\text { 1) Active and Idle handsets } \\
\text { 2) Energy Efficient } \\
\text { 3) Large sample size } \\
\text { 4) Measures average speeds }\end{array}$ & $\begin{array}{l}\text { 1) Vehicle passing boundaries of } \\
\text { cell sites with a traffic junction } \\
\text { them, encounter green or red } \\
\text { phase, which introduces error } \\
\text { 2) Depends on multiple cellular } \\
\text { service providers for } \\
\text { location detection }\end{array}$ \\
\hline $\begin{array}{l}\text { Traffic flow } \\
\text { prediction }\end{array}$ & $\begin{array}{l}\text { 1) Simulator on history data } \\
\text { 2) Real time data submitted } \\
\text { to get prediction results }\end{array}$ & $\begin{array}{l}\text { 1) Implementation not in } \\
\text { real time }\end{array}$ \\
\hline COTraMS & $\begin{array}{l}\text { 1) Employs IEEE networks } \\
\text { 2) Energy Efficient }\end{array}$ & $\begin{array}{l}\text { 1) Requires RSU installed on road } \\
\text { side making it operationally } \\
\text { challenging } \\
\text { 2) Requires IEEE } 802.11 \\
\text { infrastructure }\end{array}$ \\
\hline $\begin{array}{c}\text { SBTM } \\
\text { (Spatiotemporal } \\
\text { Traffic Bottleneck } \\
\text { Mining) }\end{array}$ & $\begin{array}{l}\text { 1) Identifies traffic bottlenecks } \\
\text { 2) Location based services } \\
\text { 3) Accuracy of Prediction > } \\
\text { (Congestion converge OR } \\
\text { Congestion drop) }\end{array}$ & $\begin{array}{l}\text { 1) Depends on multiple } \\
\text { cellular service providers } \\
\text { for location detection } \\
\text { 2) Not in real time }\end{array}$ \\
\hline $\begin{array}{c}\text { Localized Handling } \\
\text { Network Event } \\
\text { Systems } \\
\text { (LocHNESs) }\end{array}$ & $\begin{array}{l}\text { 1) Accepts fixed as well as } \\
\text { GPS enabled sensor }\end{array}$ & $\begin{array}{l}\text { 1) Large datasets } \\
\text { 2) Computation intensive } \\
\text { 3) Dependence on multiple } \\
\text { data agency }\end{array}$ \\
\hline AFRC & $\begin{array}{l}\text { 1) Network of Controllers } \\
\text { communicate } \\
\text { 2) Compute congestion levels } \\
\text { 3) Disseminates information to } \\
\text { traffic junction ahead }\end{array}$ & $\begin{array}{l}\text { 1) Physical installation of } \\
\text { controller } \\
\text { 2) Data acquisition from } \\
\text { roadside sensor is a } \\
\text { challenging hardware issue }\end{array}$ \\
\hline Real Time System & $\begin{array}{l}\text { 1) GPS Sensors } \\
\text { 2) Real time system }\end{array}$ & $\begin{array}{l}\text { 1) No inroad sensors } \\
\text { 2) No latency } \\
\text { 3) Accurate }\end{array}$ \\
\hline
\end{tabular}




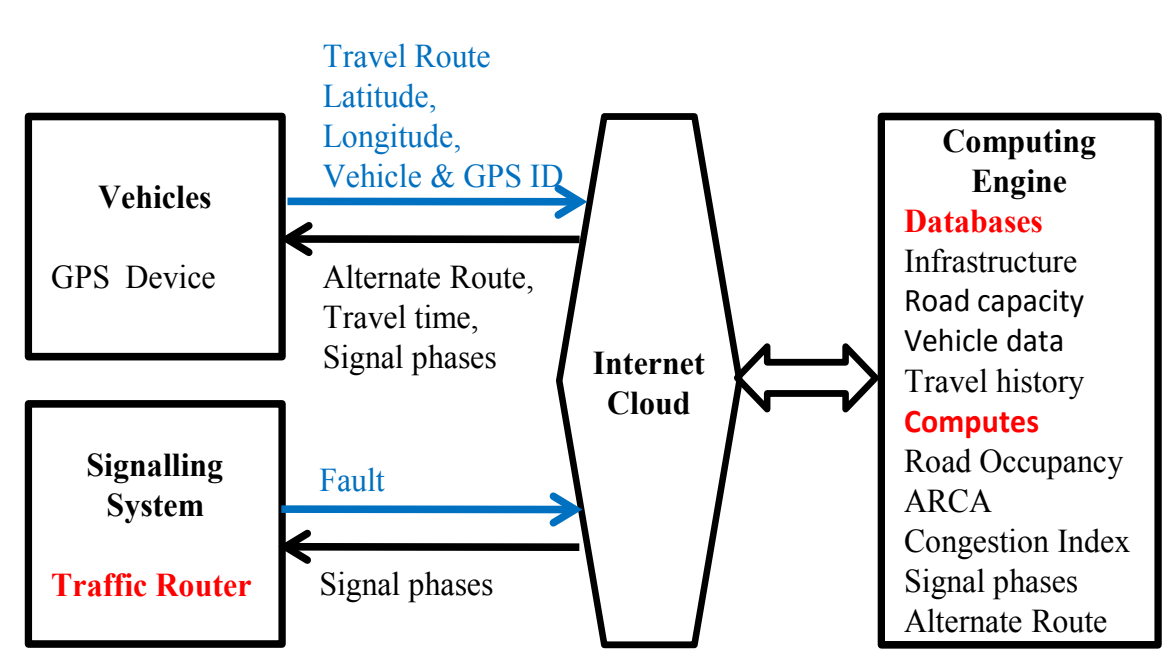

Architecture

Figure 1. Describe overall topology with cluster of vehicles, cluster of traffic routers \& traffic server.

\subsection{Road Infrastructure Database}

Road infrastructure information is required to create geographical maps, compute road capacity and average speed of the road segment. Road network has two sets of graphs: Static (distance) network graph and dynamic (congestion) network graph. The parameters stored in the database are road lengths, traffic signals at the two ends of road, number of lanes, converging smaller lanes, right of direction and quality of road. The infrastructure details are captured through a pilot vehicle equipped with an application, which automatically captures latitude and longitude with a time stamp as it travels along a road segment. The user intervenes and inserts details like number of lanes, right of way, traffic signals at the ends of the road segment, nomenclature and other converging by-lanes along the road segment without the traffic signal. The road capacity of the road segment with length $L$, number of lanes $N$ is expressed as:

$$
\mathrm{RC}=L * N
$$

\subsection{Vehicle Detection}

Every vehicle is equipped with GPS enabled device and has a unique ID. Vehicle data is entered in the traffic server database viz. vehicle registration number, vehicle manufacturer, model, length \& breadth and fuel type. Of these, vehicle size \& fuel type enables computing road occupancy and fuel consumption. Figure 2 details the functioning of in-vehicle system.

The in-vehicle unit transmits location data under program control. The transmission of location data is controlled to remove redundant data. The traffic server acquires vehicle location of all the vehicles on road and computes road occupancy of all the road segments. Vehicle speed is computed from location information with time stamp. Data logged is ingested in the data base, which builds knowledge system for travel history, travel behavior and alternate routes. 


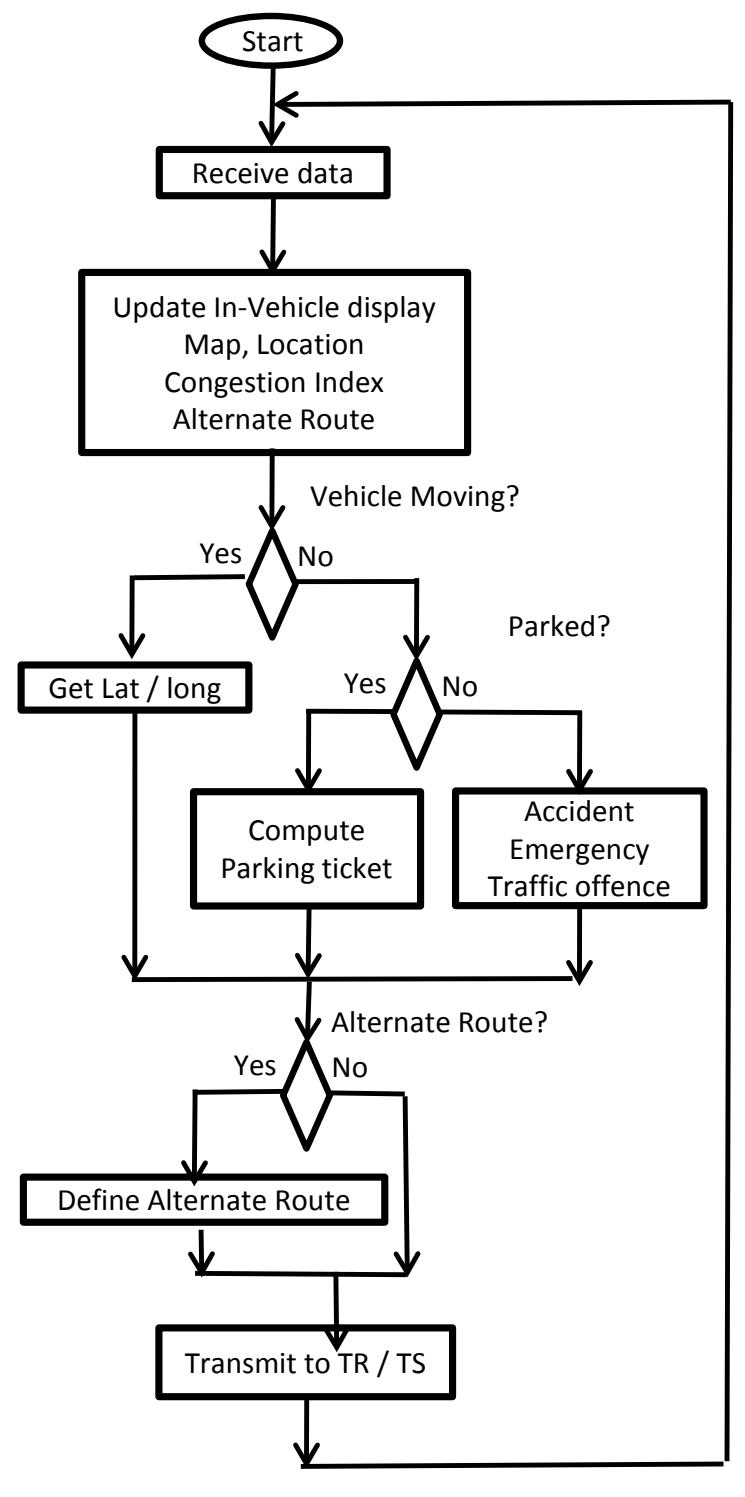

In-Vehicle

Figure 2. In-Vehicle algorithm depicts essential functionalities and deliverables of the system.

\subsection{Traffic Signal Phases-Present Scenario}

Traditionally traffic signal phases are fairly assigned to all directions, which results in wasting the green phases when there are no vehicles present in the direction assigned green phase. Secondly, the duration of green may be underutilized if fewer vehicles are there. Thirdly, when green phase is assigned to a direction, other predefined directions too gets green phase. In India, a direction gets green phase to travel left, straight and in right direction. In case there is no traffic in straight or right direction, the green phase is wasted. Ideally it must be handed over to other directions contesting for a green phase. In some of the recent strategies, traffic phases are assigned based on holidays, traffic flow, directions of traffic and peak and non-peak conditions. These are assigned manually or are 
pre-programmed and not real time allocation. The proposed strategy automatically takes care of these aspects.

\subsection{Computing Traffic Signal Phases}

The proposed strategies are:

1) Vehicle Density \& Cumulative Waiting Time (CWT)

Signal phases are assigned based on density of vehicles, waiting time of vehicles and flow rate. Directions with heavy traffic flow prevail over the other directions and acquire green phase.

$$
\tau=\sum_{1}^{N}\left(\int_{T_{1}}^{T_{2}} \mathrm{~d} t\right)
$$

$\tau=\mathrm{CWT}$ of vehicles; $T_{1}=$ Entry time of vehicle; $T_{2}=$ Time at computation; $N=$ Number of vehicles.

However, a larger waiting vehicle is assigned priority over a smaller waiting vehicle to incorporate road occupancy in terms of area. Hence the above equation is modified to:

$$
\tau=\sum_{1}^{N} A\left(\int_{T_{1}}^{T_{2}} \mathrm{~d} t\right)
$$

$A=$ Area of vehicle under consideration

If this philosophy is followed, green phase for a direction with lower CWT will be unreasonably delayed. To address this lacuna and avoid signal phase STARVATION, upper bound for a signal phase is defined. The upper bound is maximum duration of a green phase that can be assigned. To summarize:

a) The signal phase is proportional to the CWT;

b) The upper bound defines maximum time of a green phase to ensure starvation of green phase to a direction for unreasonably longer time;

c) The green phase has a lower bound to consider vehicle startup time, acceleration and time to cross the distance of traffic junction;

d) Signal phase are assigned to all directions in a single signal cycle. A traffic junction with $\mathrm{M}$ converging road segments follows the contention resolution as per the algorithm below:

Algorithm

$$
S\left(\tau_{1}, \tau_{2}, \cdots, \tau_{M}\right)
$$

For $I=1$ to $M$

Evaluate: $\operatorname{Max}\left\{S\left(\tau_{1}, \tau_{2}, \cdots, \tau_{M}\right)\right\}$;

Assign green phase

$$
S=S\left(\tau_{1}, \tau_{2}, \cdots, \tau_{M}\right)-\operatorname{Max}\left\{S\left(\tau_{1}, \tau_{2}, \cdots, \tau_{M}\right)\right\}
$$

Go to Evaluate;

End;

$S$ is a set of directions; Max computes direction with maximum CWT.

For four directions it will sequentially and cyclically compute $\operatorname{Max}\left(\tau_{1}, \tau_{2}, \tau_{3}\right.$, $\left.\tau_{4}\right)$; $\operatorname{Max}\left(\tau_{2}, \tau_{3}, \tau_{4}\right)$; $\operatorname{Max}\left(\tau_{3}, \tau_{4}\right) ; \tau_{4}$. Alternately it can be represented as Max 
(Four Directions); Max (Remaining Three Directions); Max (Remaining Two Directions); Last Remaining Direction. This will ensure no direction starves a green phase.

2) Look Ahead Buffer

Before green phase is assigned, the system ensures enough buffer is ahead to accommodate the vehicles leaving the traffic junction else it will end up in a dead lock situation. The available buffer area is considered to compute green phase so that excess vehicles are not crossing the traffic junction.

The green phase is a function of CWT and ARCA. Road Occupancy (RO) of a road segment is the area occupied by vehicles.

$$
\mathrm{RO}=\sum_{i=1}^{N} A_{i}
$$

$\mathrm{RC}$ is known from the infrastructure database, $\mathrm{RO}$ is known from the vehicles on the road segment, hence ARCA $=\mathrm{RC}-\mathrm{RO}$

$$
\mathrm{ARCA}=L * N-\sum_{i=1}^{N} A_{i}
$$

The parameters to compute are maxima of cumulative time and maxima of ARCA. It describes road conditions:
a) $\mathrm{RO}=\mathrm{ARCA} \rightarrow$ Normal traffic;
b) $\mathrm{RO}>\mathrm{ARCA} \rightarrow$ Congestion;
c) $\mathrm{RO}<\mathrm{ARCA} \rightarrow$ No congestion.

\subsection{Traffic Router/Traffic Signal Driver}

TR is co-located with traffic signals. It has the following functionalities:

1) Collect on-road vehicle coordinates;

2) Transmit collated data to traffic server (in case of multi-tier architecture);

3) Receive data from traffic server;

4) Transmit data to the vehicle to give traffic information (in case of multi-tier architecture);

5) Transmit data to the traffic display driver.

The schematic is depicted in Figure 3 and the functionality is depicted in Figure 4. All communication happens around a defined protocol.

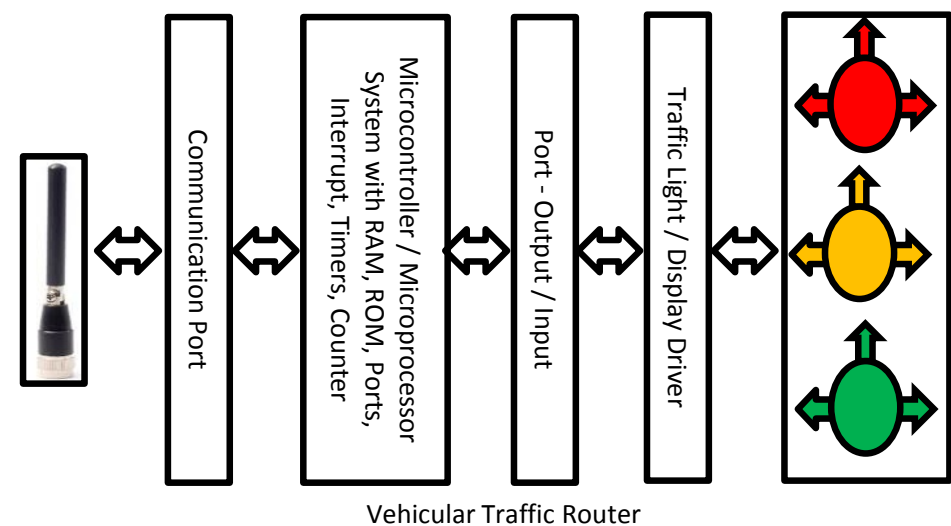

Figure 3. Traffic router. 


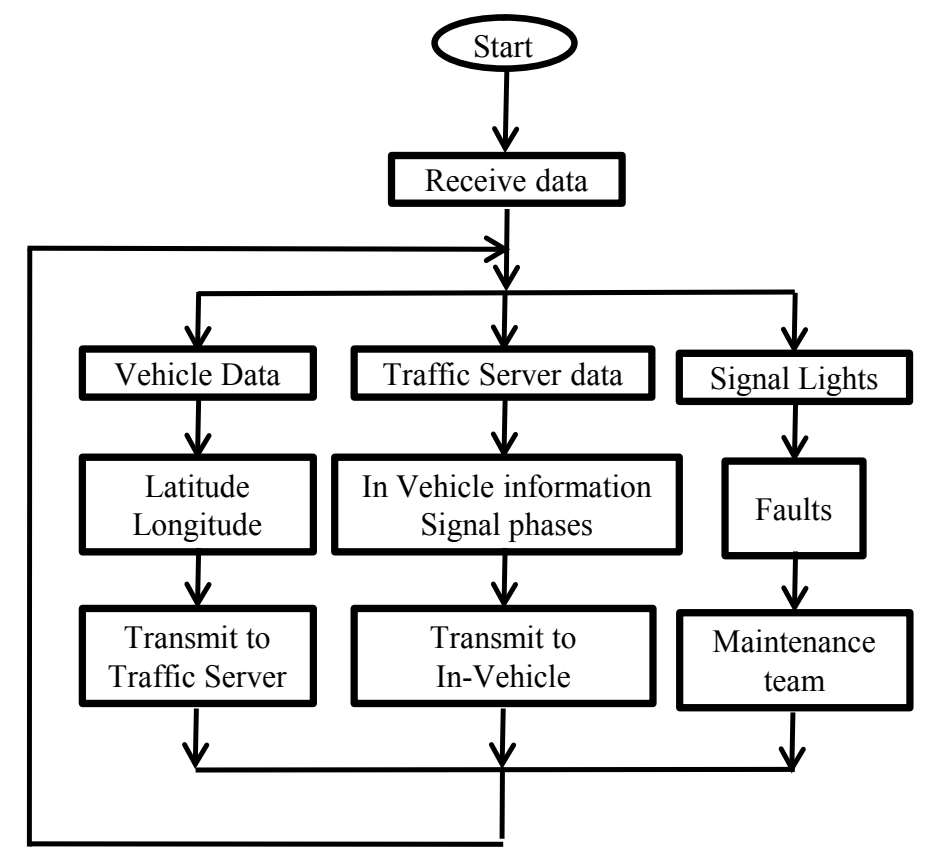

Figure 4. Traffic router algorithm is simple as it transmits and receives data under strict protocol definitions.

\subsection{Traffic Server (TS)/Computing Engine}

The algorithm is detailed in Figure 5. It has three main functions data collection, computation and data dissemination.

1) TS collate data from in-vehicle system and/or TR.

2) Compute congestion index, average speed on a road segments, road occupancy and ARCA. TS hosts and manages databases, houses infrastructure database.

3) In-vehicle system receives information regarding signal phases and congestion situation in different road segments. It also reports faults, information to planning \& maintenance teams.

\subsection{Alternate Route}

When travel time through a road segment increases several times, the congestion has reached its upper bound and hence alternate route is sought. Congestion Index (CI) defined as:

$$
\mathrm{CI}=\frac{\text { Road Occupancy }}{\text { Road Capacity }}
$$

Alternately expressed as a ratio of

$$
\mathrm{CI}=\frac{\text { Average speed of vehicles }}{\text { Rated speed }}
$$

This paper proposed a detailed solution to evaluate least cost path. Static network is a distance network and dynamic graph depends on time to travel through the road segment, which keeps on changing [9] [10]. The alternate route 


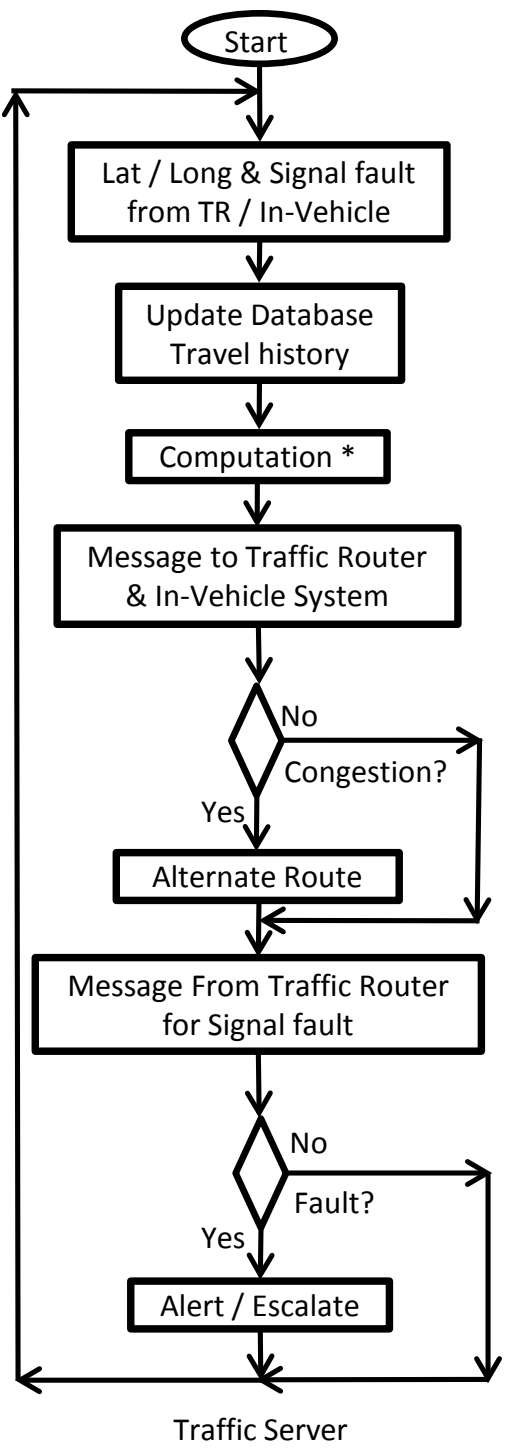

Figure 5. Traffic server communicates with in-vehicle systems as well as traffic routers. The algorithm depicts its function. Computation ${ }^{\star}$ computes Road occupancy, ARCA, congestion index, alternate routes and traffic signal phases.

is proposed based on static network, dynamic network and history data. Shortest distances from every node to other nodes is pre-computed and available as a look up table whereas dynamic network is evaluated when the edge weight changes. Travel path of every vehicle is logged and stored in the data base. This creates knowledge (History) database to refer to for preferred routes.

\section{Analyses, Results \& Conclusion}

\subsection{Analyses}

A simulator is developed in windows environment using C++ and MS SQL da- 
tabase.

1) Case 1-Single direction: Fixed signal phase

The variables set are outflow and inflow rate. Figure 6 depicts that as the outflow rate increases the congestion reduces. It also demonstrates, increasing the outflow beyond an optimum level does not yield further reduction in congestion.

2) Case 2-Traffic junction: Fixed signal phases

Applying the above to all the four directions of a junction with equal outflow, inflow and signal phases, the results are shown in Figure 7. The results are plotted for two cases viz. outflow to inflow ratio of 5:1 in part A as well as 6:1 in part B. The difference between the two is equilibrium is delayed by a cycle of signal phase in case of 5:1. In both the cases waiting vehicles swing between 0 to a maximum of 60 . Meaning once the green phase is served, it evacuates all the vehicles and while it waits next green phase, it collects 60 vehicles seeking the oncoming green phase.

3) Case 3-Traffic junction: Dynamic signal phases

During initial condition the waiting vehicles are 50, 40, 25 and 20 in north, east, west and south directions respectively. The ratio of outflow rate to inflow is 5:1. The graph is plotted in Figure 8, where the first graph is vehicles in the queue and the second graph depicts evacuating vehicles. The software assigns priority to a direction with maximum CWT. In the equilibrium position, the number of waiting vehicles stabilizes between 12 and 16 . The second graph in Figure 8 shows number of vehicles moving ahead. One can easily notice, north direction was given a green phase 6 times, followed by east 5 times, west 4.5 times and south 4 times.

On changing the initial waiting vehicles to 100 for north direction, one gets better clarity, as shown in Figure 9. Here the north direction is granted green

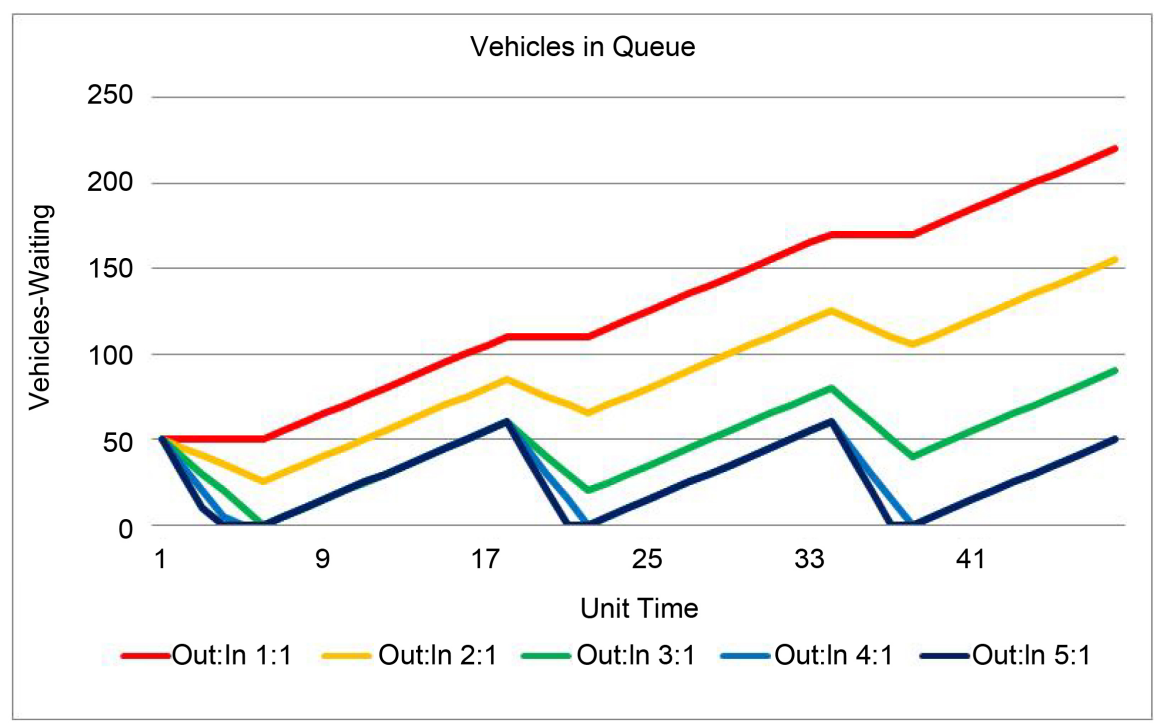

Figure 6. Graph shows vehicle accumulated at a traffic junction for different outflow to inflow ratios. 


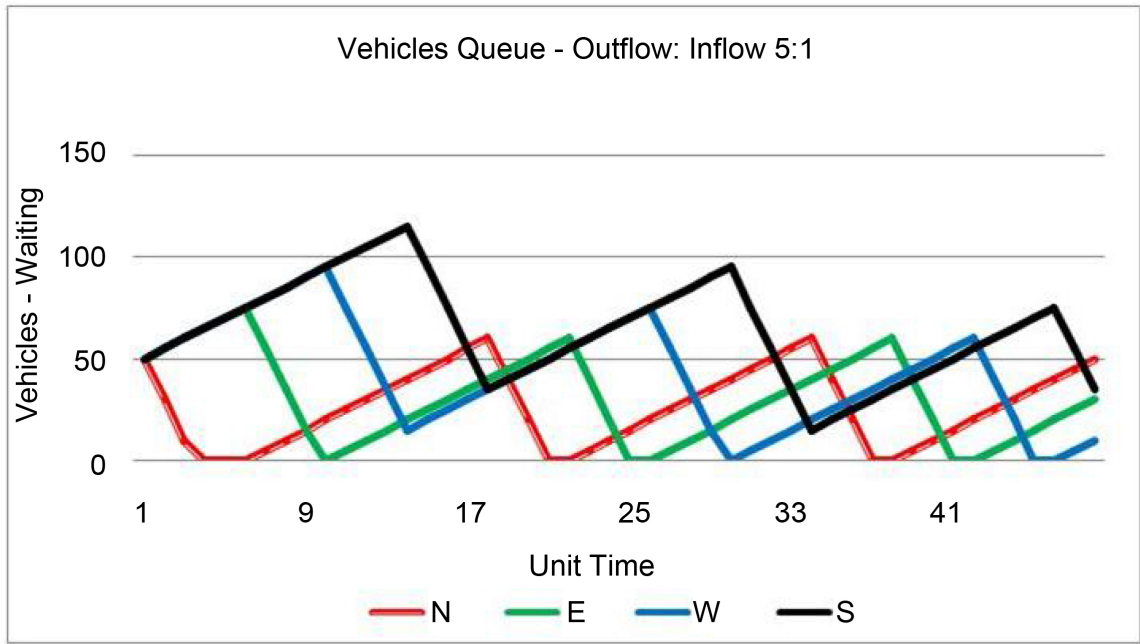

Part A

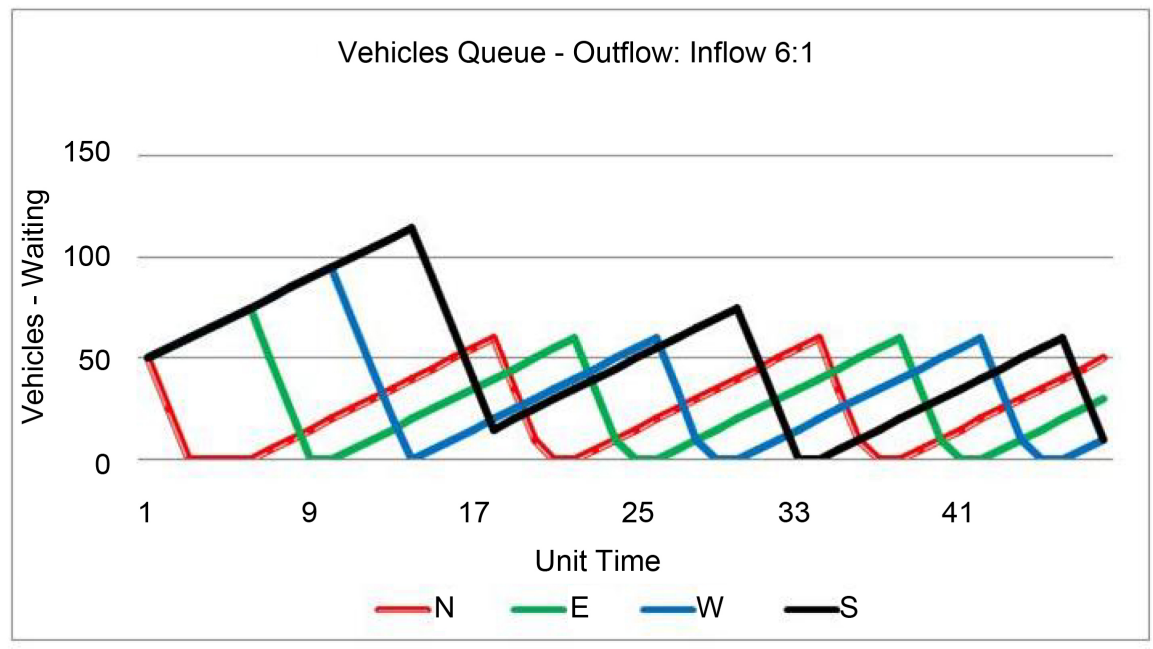

Part B

Figure 7. Graph confirms the results of case 1 albeit it takes few cycles to arrive at equilibrium.

phase six times as it had higher number of vehicles. However, this is for the initial cycles only.

\subsection{Results}

1) Employing static signaling phases the waiting vehicles vary between $0 \& 60$, average value of waiting vehicles being 30 .

2) While employing dynamic signal phases the waiting vehicles vary between $5 \& 20$, average value of waiting vehicles being 12.5 .

3) The upper limit is reduced by $66 \%$ where as the average value is $58 \%$.

4) The maximum computing cost of the proposed solution in a square matrix will be number of diagonal nodes which is diagonal distance $\sqrt{2} N$, each node having to evaluate 3 directions resulting in $3 * \sqrt{2} N$ which is $4.24^{*} N$ which is less than $N^{2}$. 


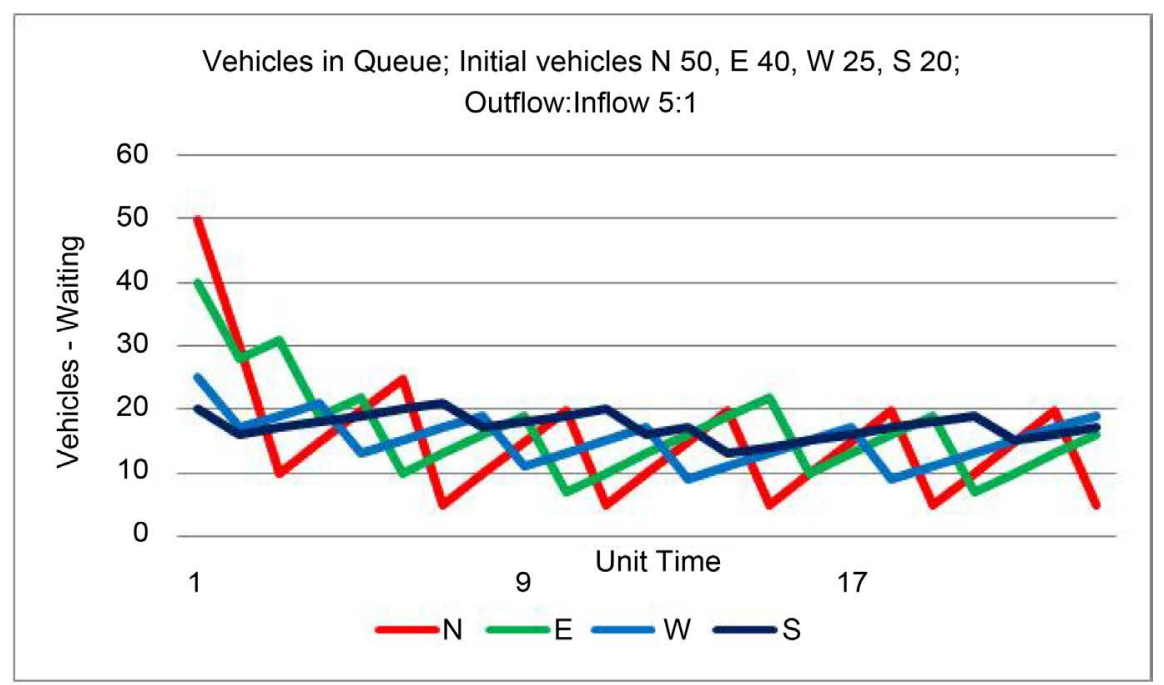

Part A

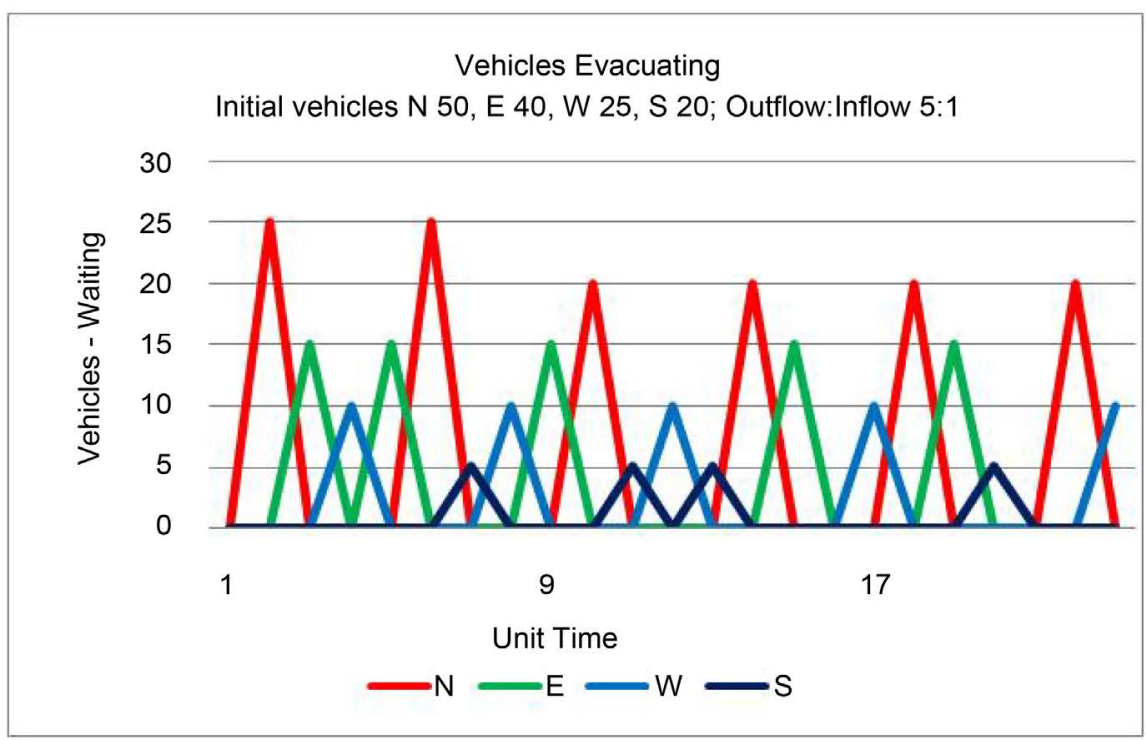

Part B

Figure 8. Graph confirms the results of case 1 albeit it takes few cycles to arrive at equilibrium.

\subsection{Conclusions}

1) In-road sensors are prohibitively costly with respect to installation, maintenance and power consumption.

2) Though GPS technology is employed, power consumption is kept to minimum by reducing GPS related query.

3) Probabilistic, analytical models are good to appreciate the severity of problem but real time implementation is the only solution.

4) Static traffic signal phases may temporarily seem to ease congestion but ultimately result in building up congestion over a period of time.

5) The exiting traffic in all the three directions is never equal and hence outflow to inflow ratio of 5:1 is the most optimized figure of merit. 


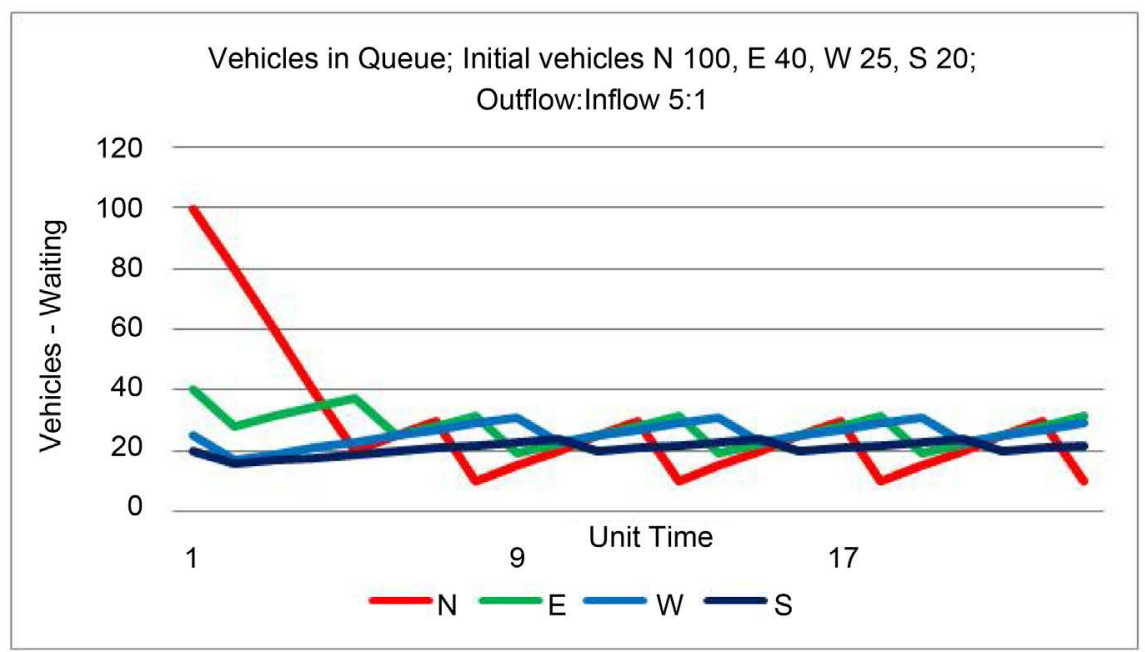

Part A

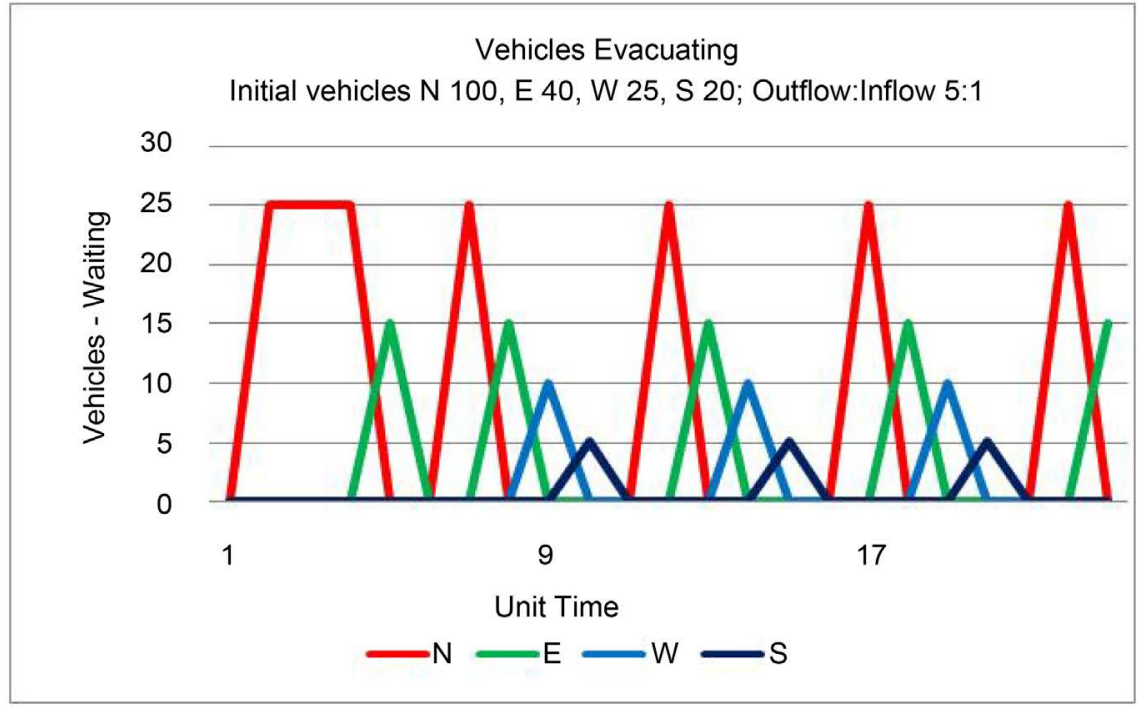

Part B

Figure 9. Graph with 100 waiting vehicles at initial condition.

6) Dynamic signaling always attains equilibrium and diffuses congestion irrespective of outflow to inflow ration. A lower ratio will take a few cycles more compared to higher outflow to inflow ratio.

7) Initially lower outflow to inflow ratio is selected to avoid wasting green phase utilization. Number of cycles of signal phases till the equilibrium is reached, deciding in favor of increasing the ratio.

8) A judicious selection of outflow to inflow ration reduces the band of minimum and maximum waiting vehicles.

9) Traffic server computation decides the outflow to inflow which ensures minimum band of waiting vehicles.

\section{Future Work}

1) Global Warming 
Vehicle travel is precisely monitored. The fuel type and fuel economy is known and hence $\mathrm{CO}_{2}$ emission is estimated accurately.

2) Traffic Signal Avoidance (TSA)

TS can send computation of signal phases to the vehicles, thus avoiding the necessity of dedicated traffic signals. It also eliminates cost of equipments, traffic router, signaling, power, costly installation and maintenance.

Proposed VICS (Vehicle-Intersection Coordination Scheme) is the one wherein the traffic signaling systems can be done away with [11]. The system coordinates through vehicular communication network to navigate traffic. The signal phase information is rendered in the vehicle. So a disciplined driving can render the traffic signal islands redundant. This paper proposes to duplicate the information of signal phases in the vehicle display, thereby rendering physical traffic junctions redundant.

3) Post Accident Analyses

In case of accident, important data, immediately before the accident like speed of the vehicle, acceleration, direction provides important insight to post accident analyses.

4) Road Quality

RQ evaluation is simplified. Average speed on a given road segment during no congestion defines the quality of the road. Comparing present day average speeds to the rated speed provides information on road quality.

5) Tax and Toll Collections

Tax collection can be done for zone based toll travel as well as pay-per-use collection. Zone based can be done since the location of travel is known all the time. Road tax can now be collected based on road usage rather than fixed charges irrespective of road usage, which will make the system fair.

6) Traffic Offenses

Traffic offenses related to jumping the traffic lights, driving in one-ways, wrong parking or long parking can be detected automatically.

\section{References}

[1] Lv, M.Q., Chen, L., Wu, X.J. and Chen, G.C. (2015) A Road Congestion Detection System Using Undedicated Mobile Phones. IEEE Transactions on Intelligent Transportation Systems, 16, 3060-3072.

[2] Janecek, A., Valerio, D., Hummel, K.A., Ricciato, F. and Hlavacs, H. (2015) The Cellular Network as a Sensor: From Mobile Phone Data to Real-Time Road Traffic Monitoring. IEEE Transactions on Intelligent Transportation Systems, 16, 2551-2572. https://doi.org/10.1109/TITS.2015.2413215

[3] Abadi, A., Rajabioun, T. and Ioannou, P.A. (2015) Traffic Flow Prediction for Road Transportation Networks with Limited Traffic Data. IEEE Transactions on Intelligent Transportation Systems, 16, 653-662.

[4] Ribeiro Jr., J.G., Campista, M.E.M. and Costa, L.H.M.K. (2014) COTraMS: A Collaborative and Opportunistic Traffic Monitoring System. IEEE Transactions on Intelligent Transportation Systems, 15, 949-958.

[5] Lee, W.-H., Tseng, S.-S., Shieh, J.-L. and Chen, H.-H. (2011) Discovering Traffic 
Bottlenecks in an Urban Network by Spatiotemporal Data Mining on Location-Based Services. IEEE Transactions on Intelligent Transportation Systems, 12, 1047-1056. https://doi.org/10.1109/TITS.2011.2144586

[6] Calabrese, F., Colonna, M., Lovisolo, P., Parata, D. and Ratti, C. (2011) Real-Time Urban Monitoring Using Cell Phones: A Case Study in Rome. IEEE Transactions on Intelligent Transportation Systems, 12, 141-151.

https://doi.org/10.1109/TITS.2010.2074196

[7] Hardy, J. and Liu, L. (2015) Reducing Vehicular Traffic Congestion Using Available forward Road Capacity Detection. IEEE International Conference on Computer and Information Technology, Ubiquitous Computing and Communications, Dependable, Autonomic and Secure Computing, Pervasive Intelligence and Computing, Liverpool, 26-28 October 2015, 2092-2097.

[8] Parmar, R.S. (2014) Identification of Parameters and Sensor Technology for Vehicular Traffic-A Survey. IJTTE International Journal of Traffic \& Transportation Engineering, 3, 101-106.

[9] Parmar, R.S. (2014) Real Time Computation of Optimal Signal Timing to Maximize Vehicular Throughput for a Traffic Junction. 3rd International Conference on Eco-Friendly Computing and Communication Systems (ICECCS 2014), Mangalore, 8-21 December 2014, 194-199.

http://ieeexplore.iee.org/xpl/login.jsp?tp=\&arnumber=7208991\&url=http\%3A\%2F \%2Fieeexplore.ieee.org\%2Fiel7\%2F7051380\%2F7208942\%2F07208991.pdf\%3Farnu mber\%3D7208991

[10] Parmar, R.S. (2016) Shortest Route-Domain Dependent, Vectored Approach to Create Highly Optimized Network for Road Traffic. IJTTE International Journal of Traffic \& Transportation Engineering, 5, 1-9.

[11] Kamal, Md.A.S., Imura, J.-I., Hayakawa, T., Ohata, A. and Aihara, K. (2015) A Vehicle-Intersection Coordination Scheme for Smooth Flows of Traffic without Using Traffic Lights. IEEE Transactions on Intelligent Transportation Systems, 16, 1136-1147. 